\title{
Modelling of Kerf Width in Plasma Jet Metal Cutting Process using ANN Approach
}

\author{
Ivan PEKO, Bogdan NEDIĆ, Aleksandar ĐORĐEVIĆ, Ivica VEŽA
}

\begin{abstract}
In this paper Artificial Neural Network (ANN) model was developed for prediction of kerf width in plasma jet metal cutting process. Process parameters whose influence was analyzed are cutting height, cutting speed and arc current. An $\mathrm{L}_{18}\left(2^{1} \times 3^{7}\right)$ Taguchi orthogonal array experiment was conducted on aluminium sheet of 3 mm thickness. Using the experimental data a feed - forward backpropagation artificial neural network model was developed. After the prediction accuracy of the developed model was verified, the model was used to generate plots that show influence of process parameters and their interactions on analzyed kerf width and to get conlusions about process parameters values that lead to minimal kerf width.
\end{abstract}

Keywords: artificial neural networks; kerf width; modelling; plasma jet metal cutting

\section{INTRODUCTION}

Today the most significant non-conventional thermal processes that can be found in different metal processing and shipbuilding industry applications are oxy fuel cutting, laser cutting and plasma jet cutting. Main advantages that characterize plasma jet cutting process are optimal combination of cut quality, productivity and costs in cutting various types of materials such as mild steel, stainless steel and aluminium at various thicknesses. Furthermore, lower investments, long consumables life and lower costs per part position this technology in front of the other cutting processes and make the company competitive.

Plasma jet cutting process is an industrial process that is essentially controlled by the operator who uses recommendations given by the manufacturers of the cutting equipment. These recommendations, however, reflect the point of view of the manufacturers' business, but do not necessarily corespond to the most acceptable solutions on the user's point of view. As a result, the user attempts to optimize the cutting operations by trial and error every time it is needed to setup the existing equipment for a new different task. This requires the development of full studies and apply theoretical and experimental researches among all the technological system's links, in order to choose the optimal processing variant.

Kumar Das et al. [1] conducted experiment on EN31 steel and investigated influence of gas pressure, arc current and torch height process parameters on responses such as material removal rate and roughness characteristics of surface cut. They used ANOVA and grey relational analysis for parametric optimization. Gullu et al. [2] investigated variations of structural specifications in heat affected zone that occur after cutting of AISI 304 stainless steel and St 52 carbon steel. Gariboldi et al. [3] analyzed cut quality in high tolerance plasma arc cutting of titanium sheets. Cut quality features that were analyzed by them are surface cut unevenness, kerf width, cut angle and surface cut roughness. They used different process conditions, plasma and shield gases and conducted temperature and microstructural investigations in the cutting edge zone. Bini et al. [4] investigated influence of process parameters like arc voltage, cutting speed, plasma gas flow rate, shield gas flow rate and shield gas composition on kerf position and shape in cutting of $15 \mathrm{~mm}$ thick mild steel plates. Salonitis et al. [5] conducted experiments on $15 \mathrm{~mm}$ thickness S235 mild steel sheets to investigate the influence of cutting speed, cutting current, cutting height and gas pressure on cut angle, surface roughness and heat affected zone. Regression analysis was used for mathematical modeling and analyzing the effect of process parameters on cut quality. Chamarthi et al. [6] conducted experiment on $12 \mathrm{~mm}$ thick plate Hardox 400 and investigated influence of process parameters like arc voltage, cutting speed and plasma gas flow rate on unevenness of cut surface. Results were analyzed through ANOVA and Design Expert software. Kavka et al. [7] analyzed the influence of plasma gas type and flow rates on kerf geometry, arc characteristics, material removal, temperature and energy in kerf zone in cutting of $15 \mathrm{~mm}$ mild steel plates. They compared response values for different gases such as steam, nitrogen, air and oxygen. Nedic et al. [8] analyzed the quality of cut in plasma arc cutting process using standard EN ISO 9013 and presented conclusions of other authors. Furthermore, they conducted experiment on steel plate of $15 \mathrm{~mm}$ thickness to investigate the influence of process parameters like arc current and cutting speed on cut quality characteristics, surface roughness, kerf width, dross width and height of molten metal on the bottom edge of the cut. Radovanovic et al. [9] developed ANN model to predict the ten point height of irregularities $(R z)$ taking input parameters such as cutting speed, cutting current and plate thickness. Developed model was used to identify cutting parameters area according to optimal surface roughness. Harničarova et al. [10] compared plasma with laser and oxygen cutting in terms of economical point of view and concluded that plasma is the most economically advantageous process. Based on their conclusions plasma cutting is 1.82 times cheaper than laser and 3.45 times cheaper than oxygen cutting process.

In the present paper experiments were made on aluminium alloy 5083 plate in order to analyze the influence of process parameters, cutting height, cutting speed and arc current on kerf width. Artificial neural network approach was used for mathematical modeling and to identify process parameters ranges that lead to optimal kerf width values. 


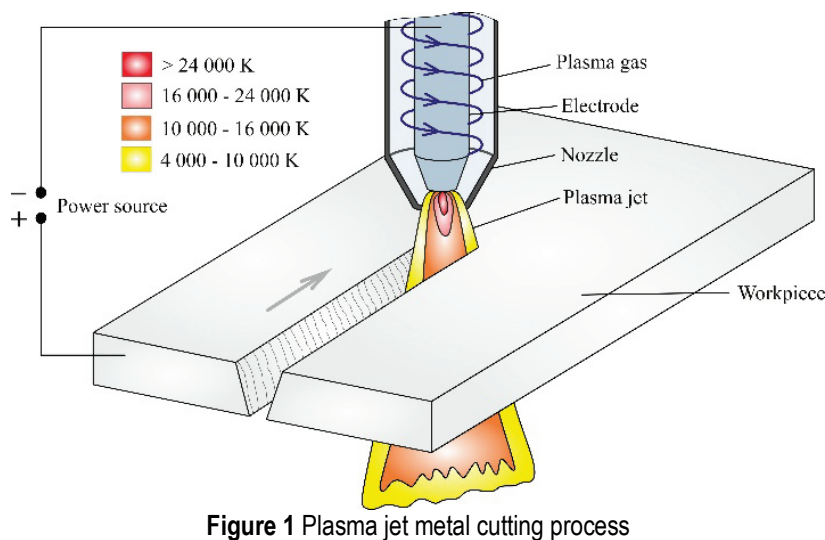

\section{EXPERIMENTAL SETUP}

All experiments were performed on a CNC FLAMECUT 2513 plasma jet cutting machine. The cuts were performed on $3 \mathrm{~mm}$ thickness Aluminium alloy 5083 plate with the use of compressed air as plasma gas. Outlet diameter of a nozzle is $1.2 \mathrm{~mm}$ and plasma gas pressure 6 bar.

Table 1 Cutting parameters and their levels

\begin{tabular}{|c|c|c|c|c|c|}
\hline No. & $\begin{array}{c}\text { Process } \\
\text { parameters }\end{array}$ & Units & Level 1 & Level 2 & Level 3 \\
\hline 1 & Cutting height, $H$ & $\mathrm{~mm}$ & 1 & 2 & - \\
\hline 2 & Cutting speed, $v$ & $\mathrm{~mm} / \mathrm{min}$ & 2000 & 4000 & 6000 \\
\hline 3 & Arc current, $I$ & A & 45 & 65 & 85 \\
\hline
\end{tabular}

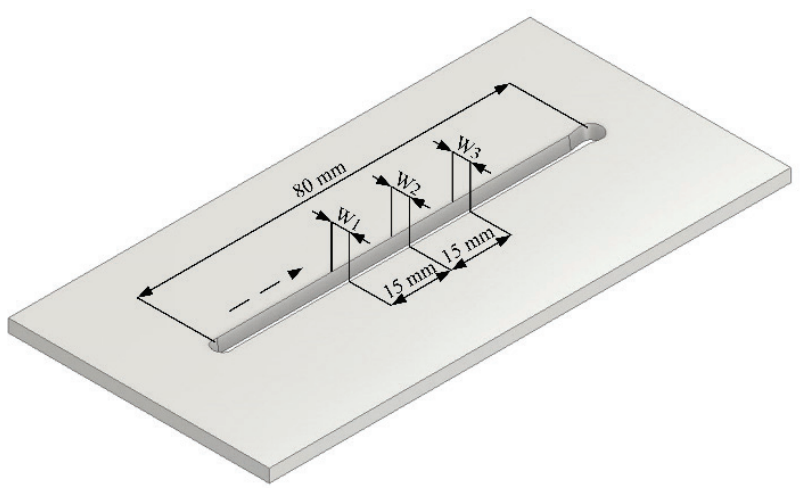

Figure 2 Look of specimen and measurement places

Table 2 Taguchi $L_{18}\left(2^{1} \times 3^{7}\right)$ orthognal array

\begin{tabular}{|c|c|c|c|c|}
\hline \multirow{2}{*}{ No. of Exp. } & \multicolumn{3}{|c|}{ Input Parameters } & Response \\
\cline { 2 - 5 } & $\begin{array}{c}\text { Cutting } \\
\text { height, } H\end{array}$ & $\begin{array}{c}\text { Cutting } \\
\text { speed, } v\end{array}$ & $\begin{array}{c}\text { Arc current, } \\
I\end{array}$ & $\begin{array}{c}\text { Kerf width, } \\
W(\mathrm{~mm})\end{array}$ \\
\hline 1 & 1 & 1 & 1 & 2.179 \\
\hline 2 & 1 & 1 & 2 & 2.058 \\
\hline 3 & 1 & 1 & 3 & 2.024 \\
\hline 4 & 1 & 2 & 1 & 1.976 \\
\hline 5 & 1 & 2 & 2 & 1.944 \\
\hline 6 & 1 & 2 & 3 & 1.993 \\
\hline 7 & 1 & 3 & 1 & 1.952 \\
\hline 8 & 1 & 3 & 2 & 2.242 \\
\hline 9 & 1 & 3 & 3 & 2.296 \\
\hline 10 & 2 & 1 & 1 & 2.185 \\
\hline 11 & 2 & 1 & 2 & 2.202 \\
\hline 12 & 2 & 1 & 3 & 2.343 \\
\hline 13 & 2 & 2 & 1 & 1.920 \\
\hline 14 & 2 & 2 & 2 & 1.957 \\
\hline 15 & 2 & 2 & 3 & 2.111 \\
\hline 16 & 2 & 3 & 1 & 1.988 \\
\hline 17 & 2 & 3 & 2 & 2.199 \\
\hline 18 & 2 & 3 & 3 & 2.492 \\
\hline
\end{tabular}

The specimens were made up of a straight cut $80 \mathrm{~mm}$ in length. All cuts were made parallel. Every experiment was conducted one time. Each measurement of kerf width was taken approximately in the middle of the cut and 15 $\mathrm{mm}$ left and right (Fig. 2). As measurement device was used a Universal Toolmaker's Microscope. Average value of all three measurements was used and traded as the result of a single experiment. Experimental results are shown in Tab. 2.

\section{ARTIFICIAL NEURAL NETWORK (ANN) 3.1 Overview of ANN}

The Artificial Neural Network represents an artificial system based on mathematical models which is, in its structure, function and information processing, similar to the biological nervous systems and, thus, able to intelligently process the information simulating the biological intelligence. The ANNs have found application in many different domains, such as dynamic system identification, complex system modeling, optimization and control, design and classification, speech interpretation, pattern recognition, metal cutting and metal forming simulation, robotics and communications and the like [11].

ModernANNs have a parallel-distributed architecture. They consist of a large number of neurons distributed in several layers. Each ANN must have three layers: input layer, hidden layer and output layer. The greatest number of ANNs has one hidden layer though there may be more of them $[12,13,14]$.

Neurons of one layer are connected by specific synapses to the neurons of their neighbouring layers. Interconnections between the neurons of the same layer do not exist. Schematically, the ANN represents an oriented graph in which the nodes are processor units, while the arrows on the lines point to the direction/sense of the signal (information) flow [15]. The interconnections between particular neurons by the layers are characterized by weights, which change during the ANN training. To better describe the relationships between input parameters and analyzed output response it is necessary to correctly adjust weights and biases in the training process.

The number of hidden layers and the number of neurons in each of them are not defined in advance: instead, these numbers can change during the ANN training until the optimum architecture is defined, namely, the one that produces the best performances of ANN. The researchers usually rely on the derived examples and their own experience ("trial and error approach"). This is one of the major obstacles in using ANNs.

The ANN modeling commonly follows these steps: definition of the input and output parameters; collection and analysis of the database; random dividing of the dataset on training, validation and testing data subsets; normalization of the input/output data (optional); designing of the ANN; training of the ANN (choice of architecture, training algorithm, transfer functions, performance criterion, and other ANN parameters); validation and testing the trained ANN.

Every ANN is used for a concrete problem. To correctly react when unknown inputs are added to it, 
artificial neural network must be trained. ANN training should be done on the examples data obtained by the experiment or some other way. Training is a process that should be repeated until the ANN is stabilized or error appears below a previously defined treshold.

The trained ANN should be validated and tested in order to asses its ability to predict and make generalization on the basis of the acquired "knowledge" on the selected set of input-output data. Validation and testing of the ANN are carried out by applying data subsets, which are not included in the training data subset. The trained, validated and tested ANN can be used for modelling and prediction of various processes.

Of all available types of ANN, multi-layer perceptron (MLP) with back propagation (BP) training procedure, is most commonly used. The back propagation ANN is designed to operate as a multilayer fully-connected feed forward network, with a particular (BP) training algorithm for supervised learning.

For feed-forward BP ANN there are many different standard training algorithms (Batch Gradient Descent, One-step-secant, Resilent Backpropagation, Conjugate Gradient, Levenberg-Marquardt (L-M), etc.). The L-M algorithm belongs to the algorithms that converge very fast (especially for smaller and medium large ANNs), with less danger from entrapment in local minimum, before reaching global minimum at error surface, while at the same time it can provide for high accuracy of prediction $[11,16,17,18,19]$.

\subsection{ANN Modeling of Plasma Jet Cutting Process}

In this paper, the three layered feed-forward back propagation ANN architecture has been used for modeling kerf width in plasma jet cutting process of alluminium alloy 5083 plate. The input layer of the ANN model consists of three neurons corresponding to the three process parameters, cutting height $(H)$, cutting speed $(v)$ and arc current $(I)$ and the output layer has one neuron for prediction of kerf width $(W)$ values (Fig. 3).

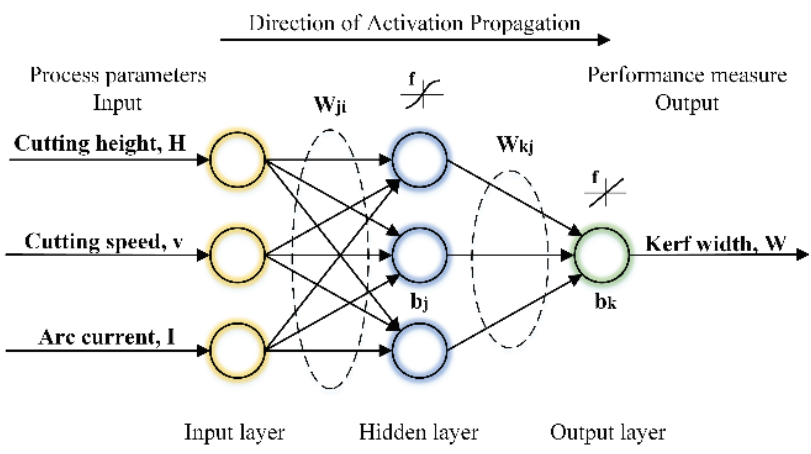

Figure 3 The selected ANN architecture for modeling plasma jet cutting process

For the needs of training, validation and testing the created ANN the whole experimental dataset $\left(N_{\text {tot }}=18\right)$ is divided into a data subset for training, a data subset for validation and a data subset for testing. 70\% randomly selected data of the whole experimental data have been employed for training, $15 \%$ for validation and $15 \%$ for testing.
Main problems that can appear in backpropagation ANNs are overfitting and overtraining. These occasions lead to a network that only memorizes and learns the training data set and relationships between known inputs and outputs but cannot generalize to new unknown data. Because of that performace of the validation data set decreases and it is necessary to find the simplest ANN model that has a prediction error considerably low. In this paper ANN architecture with one hidden layer and three neurons was used for modeling and prediction of process response values. It was found out that this architecture lead to minimal error between predicted values and real data obtained by experimental research.

Transfer functions are: tangent sigmoid function in the hidden layer and linear function in the output layer. Prior to training the training data were normalized to a range of $[-1,1]$ and the initial values of weights were set according to Nguyen-Widrow method. Back propagation algorithm with momentum was used for ANN training.

The performance of the network was measured by the Mean Squared Error (MSE) of the predicted outputs with regard to the real data obtained by experiments. The goal is to get MSE as close as possible to zero. The zero means that there is no error between outputs of the network and experimental data values. Training network was repeated until no further improvements in the MSE was achieved. It was stopped at 7 iterations. The values of MSE are given in Tab. 3 .

Table 3 MSE values for ANN model

\begin{tabular}{|c|c|c|c|}
\hline \multicolumn{4}{|c|}{ Table 3 MSE values for ANN model } \\
\hline ANN & \multicolumn{3}{|c|}{ Mean Squared Error (MSE) } \\
\cline { 2 - 4 } Architecture & Training & Validation & Testing \\
\hline $3-3-1$ & 0.0095 & 0.0182 & 0.0221 \\
\hline
\end{tabular}
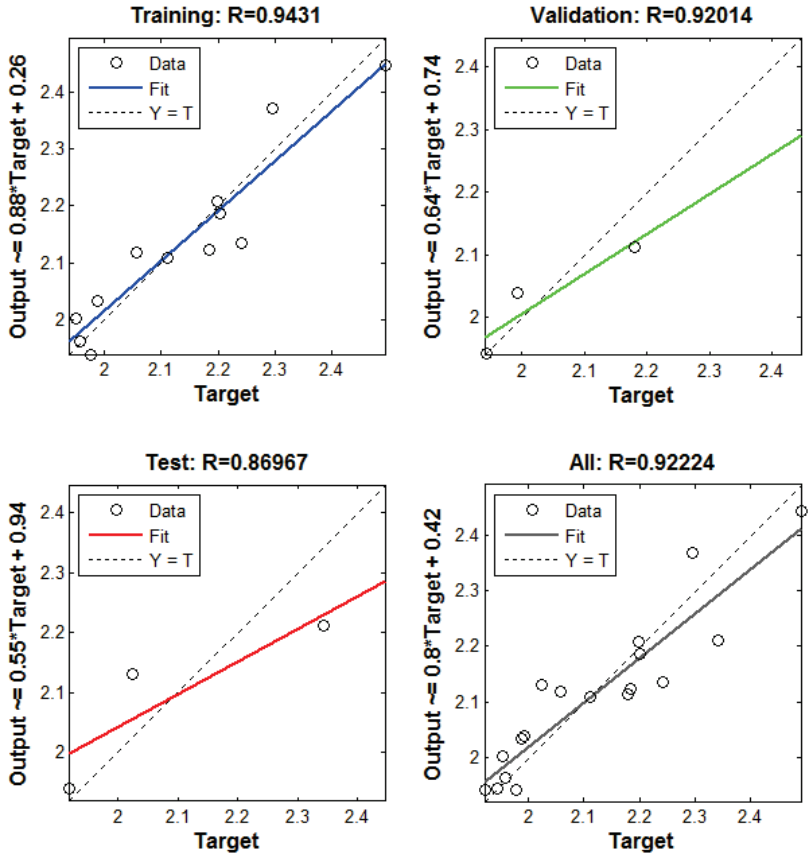

Figure 4 Correlation between predicted and experimental data in training validation and testing datasets

Except the MSE another performance measure for the process modeling by ANN approach is the correlation coefficient $(R)$. This is a statistical indicator of the strength of correlation between predicted and experimental values. A perfect correlation is obtained 
when $R=1$. A good ANN model should have the correlation coefficient greater than 0.8. Correlation coefficients of the developed ANN model for all three datasets are shown in Fig. 4.

The weights and biases of the ANN were determined during the training process and are given in Tab. 4.

Table 4 The weights and biases of developed ANN model

\begin{tabular}{|c|c|c|c|c|c|}
\hline \multicolumn{3}{|c|}{ Weights } & Weights & \multicolumn{2}{c|}{ Biases } \\
\hline-0.6382 & -0.11694 & 1.6721 & -0.31174 & 1.584 & 0.8054 \\
\hline 6.2654 & -5.1288 & -5.669 & -1.2592 & 5.5052 & \\
\hline-2.3314 & -0.68754 & 1.9069 & 0.16386 & -6.8335 & \\
\hline
\end{tabular}

Using the weights and biases from Tab. 4 the exact mathematical relationship between response, kerf width and input process parameters, cutting height, cutting speed and arc current can be expressed following the equation:

$W_{\text {norm }}=\left[\frac{2}{1+\mathrm{e}^{-2\left(X \cdot w_{j i}+b_{j}\right)}}-1\right] \cdot w_{k j}+b_{k}$

where $X$ is the column vector which contains normalized values of $H, v, I$ and $W_{\text {norm }}$ is the normalized value for the $W$. To obtain actual values for $W$ it is necessary to denormalize the data.

\section{RESULTS}

The developed ANN model was employed to analyze the effects of the plasma jet cutting process parameters on the kerf width. This was accomplished using Equation 1 and by varying the value of one or two parameters, while keeping the other parameters constant at desired level. The effects of the individual plasma jet cutting process parameters on the kerf width were represented in Figs. 5-7 while interactions of these process parameters and their influence on kerf width were shown in Figs. 8-10. The levels of parameters that were kept constant are $H=1.5$ $\mathrm{mm}, v=4000 \mathrm{~mm} / \mathrm{min}, I=65 \mathrm{~A}$.

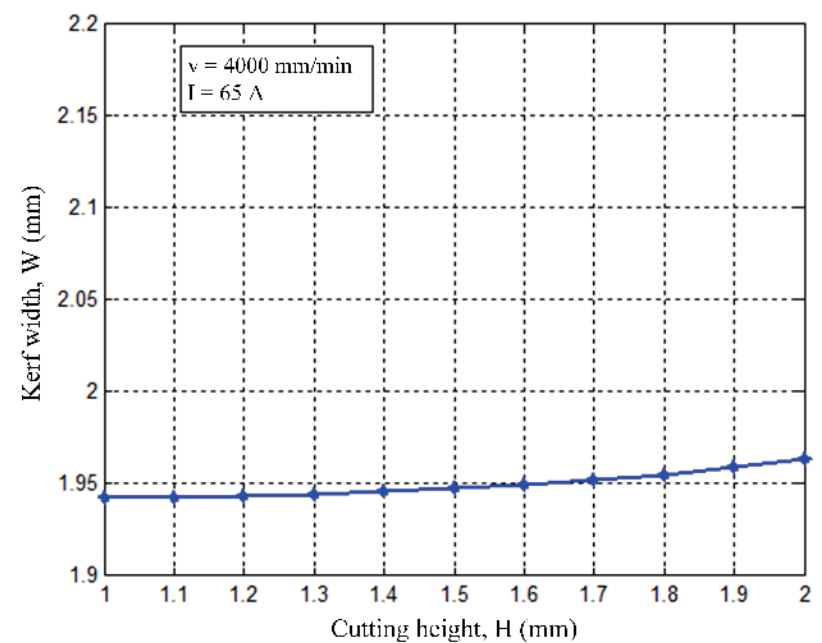

Figure 5 The influence of cutting height on the kerf width

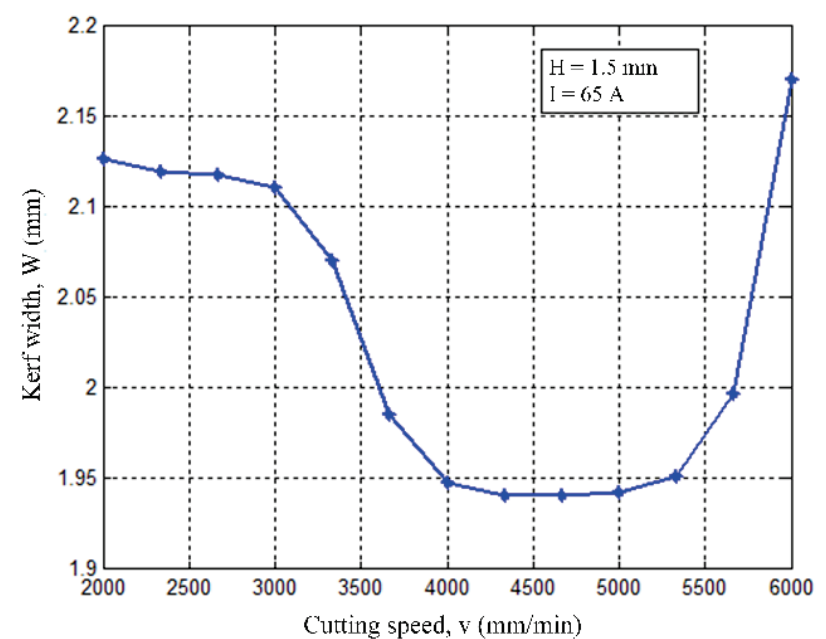

Figure 6 The influence of cutting speed on the kerf width

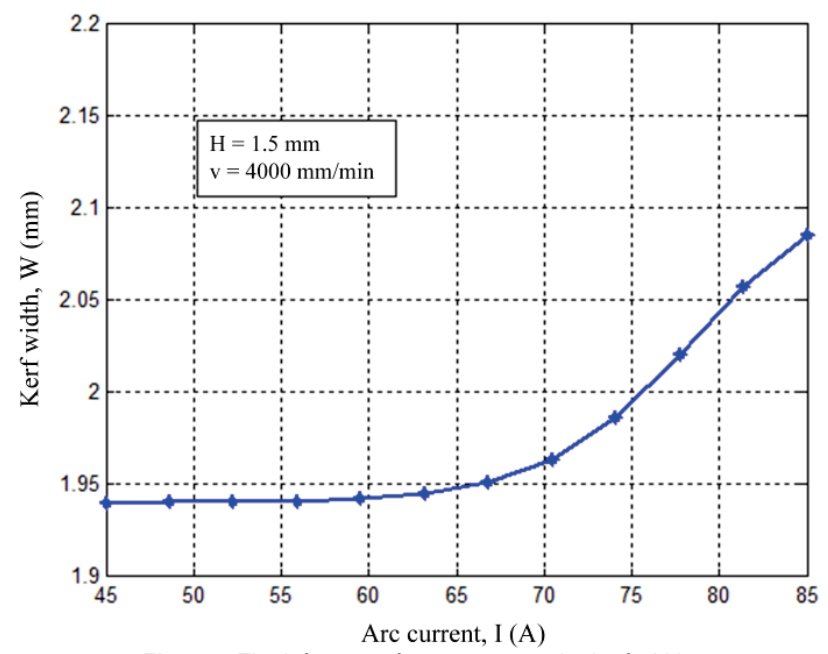

Figure 7 The influence of arc current on the kerf width

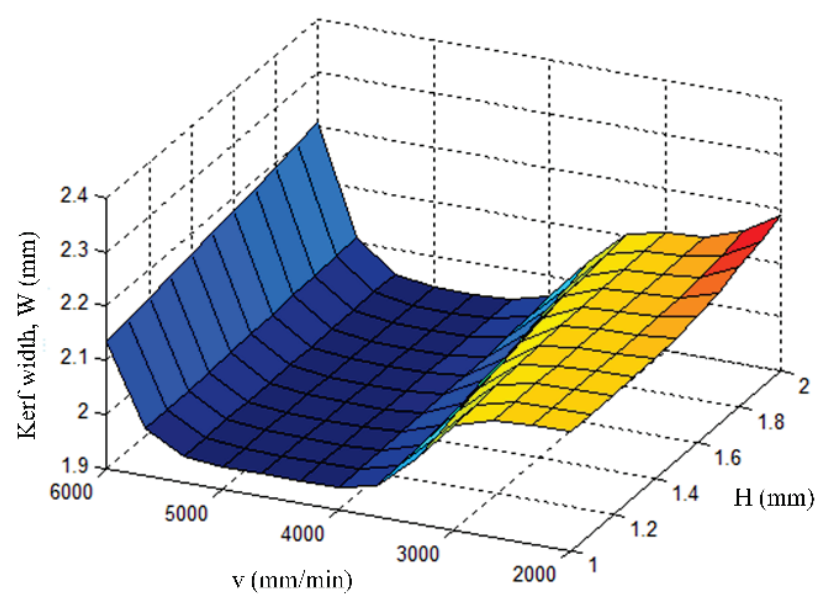

Figure 8 The influence of $\mathrm{v}-\mathrm{H}$ interaction on the kerf width

From Figs. 5-10 it can be observed that the kerf width is quite sensitive to the selected plasma jet cutting process parameters. It can be concluded that the most influential process parameters are cutting speed and arc current while cutting height has a lower influence on the analyzed kerf width. Also, it can can be seen that higher cutting speed values, from 4000 to $5000 \mathrm{~mm} / \mathrm{min}$ lead to the narrowest cut. On the other side cutting speed of 2000-3000 and $>5500 \mathrm{~mm} / \mathrm{min}$ leads to a wider cut. Likewise, an increase of the arc current at cutting speed of 2000 and $>5500$ $\mathrm{mm} / \mathrm{min}$ increases kerf width while at middle cutting 
speed values (4000-5000 $\mathrm{mm} / \mathrm{min})$ arc curent has an indistinguishable influence on kerf width increment. Cutting height has the largest influence on the kerf width on the highest arc current values and at the lowest and highest cutting speed values.

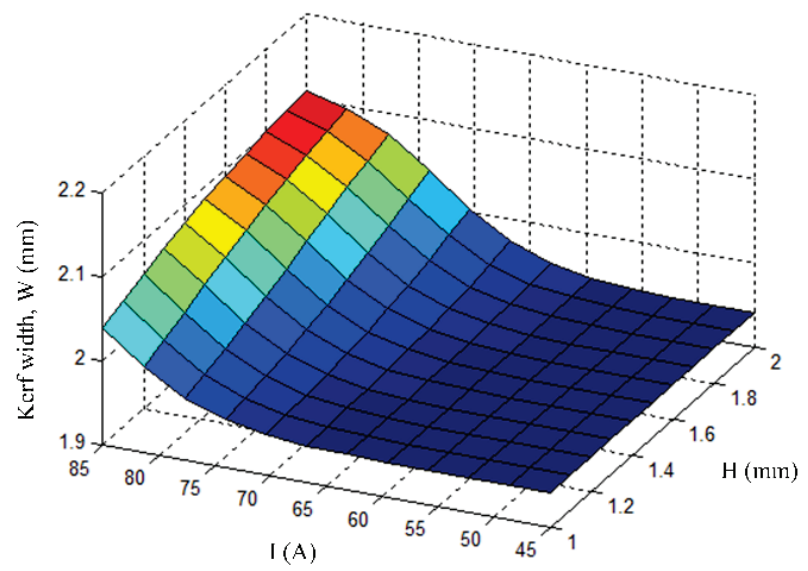

Figure 9 The influence of $\mathrm{I}-\mathrm{H}$ interaction on the kerf width

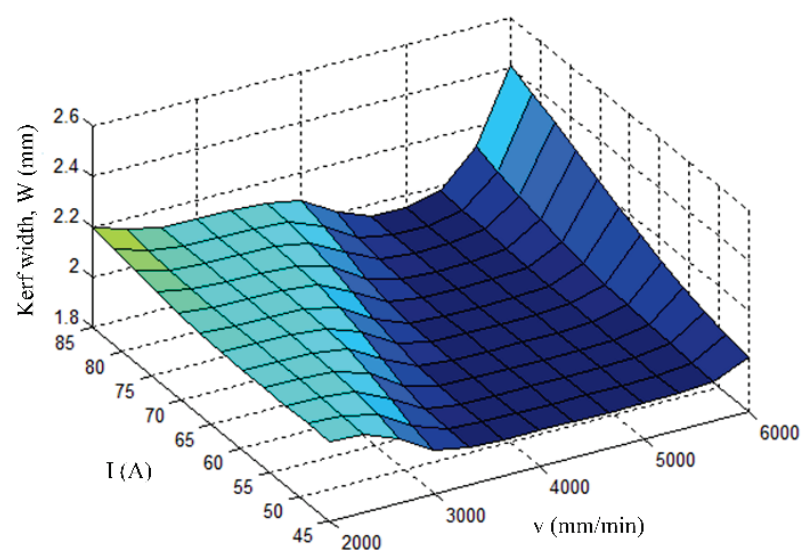

Figure 10 The influence of I-v interaction on the kerf width

\section{CONCLUSION}

In this paper mathematical modeling of kerf width in plasma jet cutting process of aluminium alloy plate was shown. Model shows dependence of kerf width on cutting height, cutting speed and arc current process parameters. Modeling was performed using three layered feedforward backpropagation neural network. Developed model was verified using statistical indicators such as MSE and R and was shown acceptable to map nonlinear inputs/output relationships. According to the ANN model the plots were generated that can be used to show process parameters influence and to identify the optimal cutting conditions which correspond to the minimal kerf width.

\section{Acknowledgements}

The paper has been fully supported by The Croatian Science Foundation under the project Innovative Smart Enterprise - INSENT (1353).

\section{REFERENCES}

[1] Kumar Das, M., Kumar, K., Barman, K. T., \& Sahu, P. (2014). Optimization of process parameters in plasma arc cutting of EN31 steel based on MRR and multiple roughness characteristics using grey relational analysis. Procedia material science, 5, 1550-1559. https://doi.org/10.1016/j.mspro.2014.07.342

[2] Gullu, A. \& Atici, U. (2006). Investigation of the effects of plasma arc parameters on the structure variation of AISI 304 and St 52 steels. Materials and Design, 27, 1157-1162. https://doi.org/10.1016/j.matdes.2005.02.014

[3] Gariboldi, E. \& Previtali, B. (2005). High tolerance plasma arc cutting of commercially pure titanium. Journal of Materials Processing Technology, 160, 77-89. https://doi.org/10.1016/j.jmatprotec.2004.04.366

[4] Bini, R., Colosimo, B. M., Kutlu, A. E., \& Monno, M. (2008). Experimental study of the features of the kerf generated by a $200 \mathrm{~A}$ high tolerance plasma arc cutting system. Journal of Materials Processing Technology, 196, 345-355. https://doi.org/10.1016/j.jmatprotec.2007.05.061

[5] Salonitis, K. \& Vatousianos, S. (2012). Experimental investigation of the plasma arc cutting process. Procedia CIRP, 3, 287-292. https://doi.org/10.1016/j.procir.2012.07.050

[6] Chamarthi, S., Reddy Sinivasa, N., Elipey Kumar, M., \& Reddy Ramana, D. V. (2013). Investigation analysis of plasma arc cutting parameters on the unevenness surface of Hardox 400 material. Procedia Engineering, 64, 854-861. https://doi.org/10.1016/j.proeng.2013.09.161

[7] Kavka, T., Mašlani, A., Hrabovsky, M., Krenek, P., Stehrer, T., \& Pauser, H. (2013). Experimental study of the effect of gas nature on plasma arc cutting of mild steel. Journal of physics D: Applied physics, 46, 1-13. https://doi.org/10.1088/0022-3727/46/22/224011

[8] Nedic, B., Jankovic, M., Radovanovic, M., \& Globocki Lakic, G. (2013). Quality of plasma cutting. $13^{\text {th }}$ International Conference on Tribology / Kragujevac, 314319.

[9] Radovanovic, M. \& Madic, M. (2011). Modeling the plasma arc cutting process using ANN. Nonconventional Technologies Review, 4, 43-48.

[10] Harničarova, M., Valiček, J., Zajac, J., Hloch, S., Čep, R., Džubakova, I., Tofil, S., Hlavaček, P., Klich, J., \& Čepova, L. (2012). Techno-economical comparison of cutting material by laser, plasma and oxygen. Technical Gazette, 19(4), 813-817.

[11] Madic, M. J. \& Marinkovic, V. J. (2010). Assesing the sensitivity of the artificial neural network to experimental noise: A case study. FME Transactions, 38(4), 189-195.

[12] Eyercioglu, O., Kanca, E., Pala, M., \& Ozbay, E. (2008). Prediction of martensite and austenite start temperatures of the Fe-based shape memory alloys by artificial neural networks. Journal of Materials Processing Technology, 200(1-3), 146-152 https://doi.org/10.1016/j.jmatprotec.2007.09.085

[13] Elangovan, K., Sathiya Narayanan, C., \& Narayanasamy, R. (2010). Modeling and forming limit diagram of perforated commercial pure aluminium sheets using artificial neural network. Computational Materials Science, 47(4), 1072-1078. https://doi.org/10.1016/j.commatsci.2009.12.016

[14] Miljković, Z., Bojović, B., \& Babić, B. (2010). Application of artificial neural network and fractals in biomedical materials surface behaviour prediction. Tehnika - Novi materijali, 19(4), 5-14.

[15] Bose, N. K. \& Liang, P. (1996). Neural network fundamentals with graphs, algorithms and applications, McGraw-Hill, New York.

[16] Haykin, S. (1998). Neural networks: A Comprehensive Foundation, Prentice Hall, New York.

[17] Dreyfus, G. (2005). Neural Networks, Springer Verlag, Berlin.

[18] Miljković, Z. (2003). Systems of Artificial Neural Networks in Production Technologies, Faculty of Mechanical 
Engineering, University of Belgrade, Belgarde, (in Serbian).

[19] Miljković, Z. \& Aleksendrić, D. (2009). Artificial Neural Networks - Collection of Resolved Tasks with Excepts From the Theory, Faculty of Mechanical Engineering, University of Belgrade, Belgarde, (in Serbian).

[20] Tzafestas, S. G., Dalianis, P. J., \& Anthopoulos, G. (1996). On the overtraining phenomenon of backpropagation neural networks. Mathematics and Computers in Simulation, 40, 507-521. https://doi.org/10.1016/0378-4754(95)00003-8

[21] Prechelt, L. (1998). Automatic early stopping using cross validation: quantifying the criteria. Neural Networks, 11(4), 761-767.

https://doi.org/10.1016/S0893-6080(98)00010-0

\section{Contact information:}

Ivan PEKO, M.Sc., Research Assistant

Faculty of Electrical Engineering, Mechanical Engineering and Naval

Architecture, University of Split,

Ruđera Boškovića 32, 21000 Split, Croatia

E-mail: ipeko@fesb.hr

Bogdan NEDIĆ, PhD, Full Professor

Faculty of Engineering, University of Kragujevac,

Sestre Janjić 6, 34000 Kragujevac, Serbia

E-mail: nedic@kg.ac.rs

\section{Aleksandar ĐORĐEVIĆ, PhD}

Faculty of Engineering, University of Kragujevac,

Sestre Janjić 6, 34000 Kragujevac, Serbia

E-mail: adjordjevic@kg.ac.rs

Ivica VEŽA, PhD, Full Professor

Faculty of Electrical Engineering, Mechanical Engineering and Naval

Architecture, University of Split,

Ruđera Boškovića 32, 21000 Split, Croatia

E-mail: iveza@fesb.hr 